\title{
SVD-Aided Beamforming and Power Allocation Algorithm for Multiuser Turbo-BLAST System Uplink with Imperfect CSI
}

\author{
Xiaomin Chen, ${ }^{1}$ Xiangbin $Y u,{ }^{1,2}$ and Dazhuan $X u^{1}$ \\ ${ }^{1}$ College of Information Science and Technology, Nanjing University of Aeronautics and Astronautics, Nanjing 210016, China \\ ${ }^{2}$ National Mobile Communications Research Laboratory, Southeast University, Nanjing 210096, China
}

Correspondence should be addressed to Xiaomin Chen, chenxm402@nuaa.edu.cn

Received 1 September 2011; Revised 10 December 2011; Accepted 14 January 2012

Academic Editor: Ananda Mohan

Copyright ( $) 2012$ Xiaomin Chen et al. This is an open access article distributed under the Creative Commons Attribution License, which permits unrestricted use, distribution, and reproduction in any medium, provided the original work is properly cited.

\begin{abstract}
The SVD-aided joint transmitter and receiver design for the uplink of CDMA-based synchronous multiuser Turbo-BLAST systems is proposed in the presence of channel state information (CSI) imperfection. At the transmitter, the beamforming and power allocation schemes are developed to maximize the capacity of the desired user. At the receiver, a suboptimal decorrelating scheme is first proposed to mitigate the multiuser interference (MUI) and decouple the detection of different users with imperfect CSI, and then the iterative detecting algorithm that takes the channel estimation error into account is designed to cancel the coantenna interference (CAI) and enhance the bit error rate (BER) results further. Simulation results show that the proposed uplink CDMA-based multiuser Turbo-BLAST model is effective, the detection from every user is completely independent to each other after decorrelating, and the system performance can be enhanced by the proposed beamforming and power allocation schemes. Furthermore, BER performance can be enhanced by the modified iterative detection. The effect of CSI imperfection is evaluated, which is proved to be a useful tool to assess the system performance with imperfect CSI.
\end{abstract}

\section{Introduction}

Multiple-input multiple-output (MIMO) communication systems provide a significant capacity increment over the conventional one through appropriate space-time processing $[1,2]$. Bell-Labs Layered Space-Time (BLAST) has shown to be a promising MIMO communication technique that can achieve tremendous bandwidth efficiencies [3]. The combination of BLAST and turbo-decoding principle is called Turbo-BLAST $[4,5]$, where the coantenna interference (CAI) caused by the major source of channel impairment can be removed by a serially concatenated iterative decoding algorithm.

Although Turbo-BLAST is considered only for singleuser application, we show herein that it naturally extends to a multiuser code division multiple access (CDMA) scenario [6], which is called CDMA-based multiuser Turbo-BLAST system, where a unique signature code is assigned to each other and used to identify the user and to spread the spectrum of the user's data. Since all the users "simultaneously" occupy the same spectrum, they create multiuser interference (MUI) to one another because of the nonzero cross correlation of their signatures [7, 8]. The MUI not only limits the system capacity but also increases the system error performance. One of the most promising approaches to meet these challenges is the use of adaptive beamforming and power control [9-13].

In the uplink (UL) of CDMA-based multiuser TurboBLAST systems, both the multiple MUI and CAI have to be mitigated. Adaptive interference suppression techniques based on multiuser detection (MUD) and antenna array processing have recently been considered as powerful methods for increasing the quality, capacity, and coverage of these systems. However, the method of optimal MUD has a very high computation cost due to its nonlinear nature [14-16].

Moreover, the performance of multiuser Turbo-BLAST system is closely related to the channel state information (CSI). Practically, CSI at the receiver is subject to the error performance because of the non-real-time data processing, quantization error, and imperfect channel estimations, and so forth [17]. This gives rise to significant challenges to system design and analysis. 


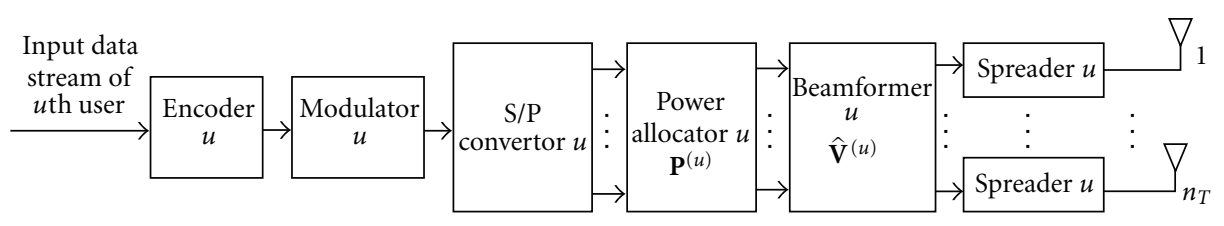

Figure 1: Transmitter structure of the $u$ th mobile station.

Regarding these problems, the SVD-assisted beamforming and power allocation schemes are first developed to maximize the capacity of every active user, and a suboptimal decorrelating scheme for the uplink of multiuser TurboBLAST system is proposed to mitigate the MUI, then the detection is performed independently for different users after decorrelating. Based on this, an improved iterative detection algorithm is adopted to cancel the CAI. We assume that the spreading structures of all the users are perfectly known to both the transmitter and the receiver at the instant of transmission and reception, and the channel estimation is imperfect, only the channel estimation matrix and the statistical characteristic of channel estimation error are known to the system. Besides, in this paper, the common assumption that true channel matrix and channel estimation error are complex Gaussian is employed, and a CDMA Frequency Division Duplexing (FDD) system is considered.

Based on the above analysis, in this paper, we derive a beamforming and power allocation algorithm for multiuser Turbo-BLAST system in the presence of imperfect CSI, which avoids that most of the existing schemes are based on perfect CSI and designed for single-user case. Moreover, by using the obtained beamforming and power allocation, an improved iterative detection scheme is also presented. With this detection scheme, the system performance is improved further. Simulation results verify the validity of the presented schemes.

This paper is organized as follows: after the introduction in Section 1, the basic system is briefly described in Section 2 . Section 3 presents an equivalent model for multiuser TurboBLAST system, the SVD-aided beamforming and power allocation scheme, and a suboptimal decorrelating algorithm, as well as the iterative detecting method which are described respectively. The simulation results are analyzed in Section 4, and Section 5 makes the conclusion of the paper.

\section{Channel Model and Basic System Description}

2.1. Transmitter Model. We consider an uplink (UL) of synchronous CDMA-based multiuser Turbo-BLAST system with $U$ active users operating in a frequency-nonselective Rayleigh fading channel, that is, a narrowband multiuser Turbo-BLAST system is considered, where CDMA is mainly employed as a multiple access technique to differentiate different users and the corresponding spreading gain is not considered. At the transmitter, a data stream of each user is first encoded, mapped into a symbol stream that is subsequently demultiplexed into $n_{T}$ substreams, and then preprocessed by the right singular matrix, of channel estimation matrix, where each stream is allocated with proper power, finally the preprocessed streams are spread and transmitted by the MS each of which employs $n_{T}$ transmit antennas. The schematic transmit structure of the $u$ th $(u=1,2, \ldots, U)$ mobile station (MS) is shown in Figure 1, where $\widehat{\mathbf{V}}^{(u)}$ represents the transmitter's beamforming matrix formulated for the transmission of the $u$ th MS's data and $\mathbf{P}^{(u)}$ is the power allocation matrix for the $u$ th MS under the constrain of total transmit power.

2.2. Imperfect Channel Model and Singular Value Decomposition. Consider a multiuser Turbo-BLAST system where the base station (BS) employs $n_{R}$ antennas and each user is equipped with $n_{T}$ antennas $\left(n_{T} \leq n_{R}\right)$. We introduce an imperfect channel model in this section [17]. For the $u$ th user, let $\mathbf{H}^{(u)} \in \mathbb{C}^{n_{R} \times n_{T}}$ denote the true complex channel matrix and $\hat{\mathbf{H}}^{(u)} \in \mathbb{C}^{n_{R} \times n_{T}}$ denote the complex channel estimation matrix. Note that the channel estimation matrix and the true channel matrix are different from one another for the uplink. Based on the imperfect channel model, the true complex matrix $\mathbf{H}^{(u)}$ can be formulated as

$$
\mathbf{H}^{(u)}=\hat{\mathbf{H}}^{(u)}+\mathbf{\Xi}^{(u)},
$$

where $\boldsymbol{\Xi}^{(u)} \in \mathbb{C}^{n_{R} \times n_{T}}$ is a complex matrix related to the imperfect CSI for the $u$ th user. The true channel matrix $\mathbf{H}^{(u)}$ and channel estimation errors $\boldsymbol{\Xi}^{(u)}$ are complex Gaussian, which leads the estimated channel matrix $\hat{\mathbf{H}}^{(u)}$ to be also complex Gaussian. Hence their statistical distributions are shown as $e_{i j}^{(u)} \sim \mathcal{C} \mathcal{N}\left(0, \sigma_{e}^{2}\right), \hat{h}_{i j}^{(u)} \sim \mathcal{C} \mathcal{N}\left(0,1-\sigma_{e}^{2}\right)$, and $h_{i j}^{(u)} \sim$ $\mathcal{C} \mathcal{N}(0,1)$, which are all distributed by complex Gaussian law, and $\sigma_{e}^{2}$ indicates the inaccuracy of the CSI.

Since the practical system cannot detect the degree of the imperfection of the CSI, the channel estimation matrix $\hat{\mathbf{H}}^{(u)}$ is treated as the true channel matrix, which can be decomposed as

$$
\hat{\mathbf{H}}^{(u)}=\widehat{\boldsymbol{\Phi}}^{(u)} \hat{\boldsymbol{\Lambda}}^{(u)}\left[\hat{\mathbf{V}}^{(u)}\right]^{H},
$$

where $(\cdot)^{H}$ denotes a matrix conjugate transpose, $\hat{\boldsymbol{\Phi}}^{(u)}$ and $\hat{\mathbf{V}}^{(u)}$ are the unitary matrices with left and right singular vectors of $\hat{\mathbf{H}}^{(u)}$ as their columns, $\hat{\Lambda}^{(u)}$ is a nonnegative and diagonal matrix, $\left\{\sqrt{\hat{\boldsymbol{\lambda}}_{i}}\right\}_{i=1}^{n_{T}}$ are the main diagonal elements of $\hat{\boldsymbol{\Lambda}}^{(u)}$, and $\left\{\hat{\boldsymbol{\lambda}}_{i}^{(u)}\right\}_{i=1}^{n_{T}}$ are eigenvalues of $\hat{\mathbf{H}}^{(u)}\left[\hat{\mathbf{H}}^{(u)}\right]^{H}$, respectively.

2.3. Multiuser Receiver Description. The illustration diagram of the receiver is shown in Figure 2, where the $u$ th user is assumed as the desired user. The decorrelating scheme and 


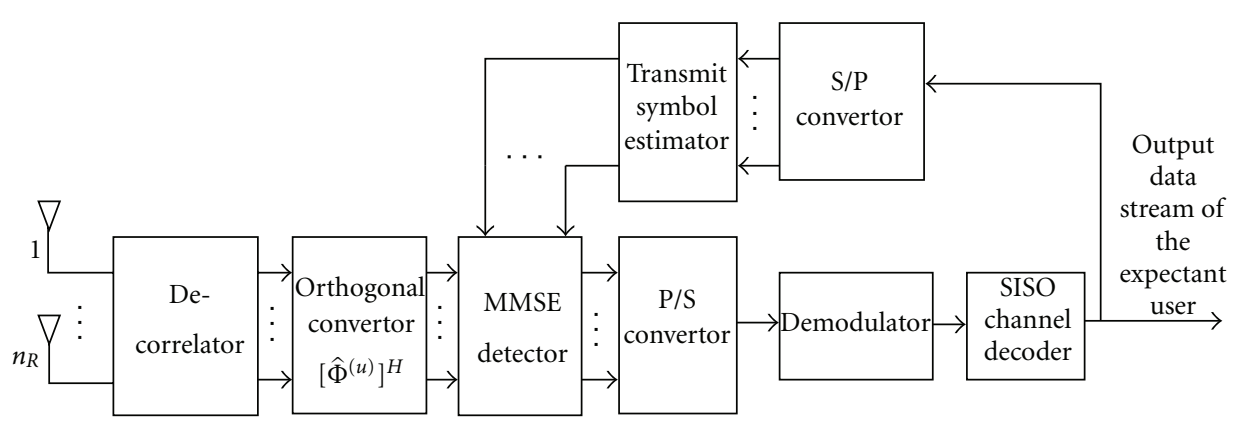

Figure 2: The receiver structure of the multiuser Turbo-BLAST system.

the iterative detection algorithm are derived based on the availability of the spreading structure of both the desired and interfering users, as well as the CSI of the desired user.

At the receiver, the received signal is decorrelated and then the signal of the desired user can be extracted after decorrelating through the use of the orthogonal spreading codes. The decoupled signal is orthogonally converted by $\widehat{\boldsymbol{\Phi}}^{(u)}$, the left singular matrix of $\hat{\mathbf{H}}^{(u)}$. Finally an iterative detection strategy based on the "turbo" principles is used for the symbol detection after orthogonally converting. For the iterative decoding, the optimal decoding process can be separated into two stages: soft-input/soft-output (SISO) channel detector and SISO channel decoder, which mutually exchange the extrinsic information sent from one stage to the other iteratively until the decoding process converges.

Let $n_{T}$ data streams be transmitted by the $u$ th MS to the BS hosted by a vector expressed as $\mathbf{x}^{(u)}=\left[x_{1}^{(u)}, \ldots\right.$, $\left.x_{n_{T}}^{(u)}\right]^{T}, u=1,2, \ldots, U,\left\{s^{(u)}(l)\right\}_{l=1}^{L}$ denotes the spreading codes of the length $L$ assigned to the desired user, and $\mathbf{n}(l)=\left[n_{1}(l), \ldots, n_{n_{T}}(l)\right]^{T}$ is a column vector of additive Gaussian noise variables during the $l$ th epoch, where each component is independent and identically distributed (i.i.d) zero-mean complex Gaussian variables with the variance of $\sigma_{n}^{2}$. Under these assumptions, the baseband discrete-time signal received by $n_{R}$ receive antennas at the BS can be expressed as

$$
\mathbf{y}(l)=\sum_{u=1}^{U} \mathbf{H}^{(u)} \hat{\mathbf{V}}^{(u)} \mathbf{P}^{(u)} \mathbf{x}^{(u)} s^{(u)}(l)+\mathbf{n}(l),
$$

where $\mathbf{P}^{(u)}=\operatorname{diag}\left(\sqrt{P_{1}^{(u)}}, \ldots, \sqrt{P_{n_{T}}^{(u)}}\right)$ is the diagonal transmit power matrix for the desired user with total power constraint $\sum_{k=1}^{n_{T}} P_{k}^{(u)}=P_{\text {total }}=n_{T}$.

\section{Equivalent System Model and Receiver Design}

3.1. Equivalent System Model. In this subsection, an equivalent system model is proposed for the uplink of the multiuser Turbo-BLAST system with imperfect CSI.
For a synchronous multiuser Turbo-BLAST system, at a sampling instant, the received signal can be formulated as

$$
\begin{aligned}
\mathbf{y}(l)= & \sum_{u=1}^{U}\left[\hat{\mathbf{H}}^{(u)}+\boldsymbol{\Xi}^{(u)}\right] \hat{\mathbf{V}}^{(u)} \mathbf{P}^{(u)} \mathbf{x}^{(u)} s^{(u)}(l)+\mathbf{n}(l) \\
= & \sum_{u=1}^{U} \hat{\mathbf{H}}^{(u)} \hat{\mathbf{V}}^{(u)} \mathbf{P}^{(u)} \mathbf{x}^{(u)} s^{(u)}(l) \\
& +\sum_{u=1}^{U} \boldsymbol{\Xi}^{(u)} \hat{\mathbf{V}}^{(u)} \mathbf{P}^{(u)} \mathbf{x}^{(u)} s^{(u)}(l)+\mathbf{n}(l) \\
= & \sum_{u=1}^{U} \hat{\mathbf{H}}^{(u)} \hat{\mathbf{V}}^{(u)} \mathbf{P}^{(u)} \mathbf{x}^{(u)} s^{(u)}(l)+\widehat{\mathbf{n}}(l)
\end{aligned}
$$

where

$$
\widehat{\mathbf{n}}(l) \triangleq \sum_{u=1}^{U} \boldsymbol{\Xi}^{(u)} \hat{\mathbf{V}}^{(u)} \mathbf{P}^{(u)} \mathbf{x}^{(u)} s^{(u)}(l)+\mathbf{n}(l),
$$

$\widehat{\mathbf{n}}(l)$ is the equivalent additive noise at the $l$ th instant, which is consisted of the interference caused by the channel estimation errors of all the interfering users and the complex Gaussian noise, respectively.

The variance of the equivalent noise $\widehat{\mathbf{n}}(l)$ is calculated as follows.

First, let $\mathbf{G}^{(u)}=\mathbf{\Xi}^{(u)} \hat{\mathbf{V}}^{(u)}$; the mean and variance of $\mathbf{G}^{(u)}$ can be evaluated as

$$
\begin{aligned}
\varepsilon\left\{\mathbf{G}^{(u)}\right\} & =\varepsilon\left\{\boldsymbol{\Xi}^{(u)} \hat{\mathbf{V}}^{(u)}\right\}=\varepsilon\left[\boldsymbol{\Xi}^{(u)}\right] \hat{\mathbf{V}}^{(u)}=\mathbf{0}, \\
\varepsilon\left\{\mathbf{G}^{(u)}\left[\mathbf{G}^{(u)}\right]^{H}\right\} & =\varepsilon\left\{\boldsymbol{\Xi}^{(u)} \hat{\mathbf{V}}^{(u)}\left[\hat{\mathbf{V}}^{(u)}\right]^{H}\left[\boldsymbol{\Xi}^{(u)}\right]^{H}\right\} \\
& =\varepsilon\left\{\boldsymbol{\Xi}^{(u)}\left[\boldsymbol{\Xi}^{(u)}\right]^{H}\right\} \\
& =\sigma_{e}^{2} \mathbf{I}_{n_{R}} .
\end{aligned}
$$

Clearly, the components of $\mathbf{G}^{(u)}$ are i.i.d zero-mean complex Gaussian variables with the variance of $\sigma_{e}^{2}$, thus statistical 
distributions can be expressed as $g_{i j}^{(u)} \sim \mathcal{C} \mathcal{N}\left(0, \sigma_{e}^{2}\right)$ and $\varepsilon\left\{g_{k i}^{(u)}\left[g_{k i^{\prime}}^{\left(u^{\prime}\right)}\right]\right\}=\sigma_{e}^{2} \delta\left(i-i^{\prime}\right) \delta\left(u-u^{\prime}\right)$.

Then, let $\mathbf{f}(l)=\sum_{u=1}^{U} \mathbf{G}^{(u)} \mathbf{P}^{(u)} \mathbf{x}^{(u)} \mathcal{s}^{(u)}(l)$; the mean and variance of $\mathbf{f}(l)$ can be evaluated as

$$
\begin{aligned}
& \varepsilon[\mathbf{f}(l)]=\varepsilon\left[\sum_{u=1}^{U} \mathbf{G}^{(u)} \mathbf{P}^{(u)} \mathbf{x}^{(u)} s^{(u)}(l)\right] \\
& =\sum_{u=1}^{U} \varepsilon\left[\mathbf{G}^{(u)}\right] \mathbf{P}^{(u)} \mathbf{x}^{(u)} s^{(u)}(l)=\mathbf{0}, \\
& \mathbf{f}(l)=\sum_{u=1}^{U} \mathbf{G}^{(u)} \mathbf{P}^{(u)} \mathbf{x}^{(u)} s^{(u)}(l) \\
& =\sum_{u=1}^{U}\left\{\left[\begin{array}{ccccc}
g_{11}^{(u)} & \cdots & g_{1 k}^{(u)} & \cdots & g_{1 n_{T}}^{(u)} \\
\vdots & \ddots & \vdots & \ddots & \vdots \\
g_{k 1}^{(u)} & \cdots & g_{k k}^{(u)} & \cdots & g_{k n_{T}}^{(u)} \\
\vdots & \ddots & \vdots & \ddots & \vdots \\
g_{n_{R} 1}^{(u)} & \cdots & g_{n_{R} k}^{(u)} & \cdots & g_{n_{R} n_{T}}^{(u)}
\end{array}\right]\right. \\
& \times\left[\begin{array}{ccccc}
\sqrt{P_{1}^{(u)}} & \cdots & 0 & \cdots & 0 \\
\vdots & \ddots & \vdots & \ddots & \vdots \\
0 & \cdots & \sqrt{P_{k}^{(u)}} & \cdots & 0 \\
\vdots & \ddots & \vdots & \ddots & \vdots \\
0 & \cdots & 0 & \cdots & \sqrt{P_{n_{T}}^{(u)}}
\end{array}\right] \\
& \left.\times\left[\begin{array}{c}
x_{1}^{(u)} s^{(u)}(l) \\
\vdots \\
x_{k}^{(u)} s^{(u)}(l) \\
\vdots \\
x_{n_{T}}^{(u)} s^{(u)}(l)
\end{array}\right]\right\}
\end{aligned}
$$$$
=\left[\begin{array}{c}
\sum_{u=1}^{U} \sum_{i=1}^{n_{T}} g_{1 i}^{(u)} \sqrt{P_{i}^{(u)}} x_{i}^{(u)} s^{(u)}(l) \\
\vdots \\
\sum_{u=1}^{U} \sum_{i=1}^{n_{T}} g_{k i}^{(u)} \sqrt{P_{i}^{(u)}} x_{i}^{(u)} s^{(u)}(l) \\
\vdots \\
\sum_{u=1}^{U} \sum_{i=1}^{n_{T}} g_{n_{R} i}^{(u)} \sqrt{P_{i}^{(u)}} x_{i}^{(u)} s^{(u)}(l)
\end{array}\right]=\left[\begin{array}{c}
f_{1}(l) \\
\vdots \\
f_{k}(l) \\
\vdots \\
f_{n_{R}}(l)
\end{array}\right]
$$

Clearly, $\mathbf{f}(l)$ is a zero-mean complex Gaussian column vector with its component $f_{k}(l)=$ $\sum_{u=1}^{U} \sum_{i=1}^{n_{T}} g_{k i}^{(u)} \sqrt{P_{i}^{(u)}} x_{i}^{(u)} s^{(u)}(l), \quad k=1, \ldots, n_{R}, l=1, \ldots, L_{S}$, whose variance can be evaluated as

$$
\begin{aligned}
\sigma_{f_{k}}^{2}(l)=\varepsilon\{ & {\left.\left[f_{k}(l)\right]\left[f_{k}(l)\right]^{*}\right\} } \\
=\varepsilon & \left\{\left[\sum_{u=1}^{U} \sum_{i=1}^{n_{T}} g_{k i}^{(u)} \sqrt{P_{i}^{(u)}} x_{i}^{(u)} s^{(u)}(l)\right]\right. \\
& \left.\times\left[\sum_{u^{\prime}=1}^{U} \sum_{i^{\prime}=1}^{n_{T}} g_{k i^{\prime}}^{\left(u^{\prime}\right)} \sqrt{P_{i^{\prime}}^{\left(u^{\prime}\right)}} x_{i^{\prime}}^{\left(u^{\prime}\right)} s^{\left(u^{\prime}\right)}(l)\right]^{*}\right\} .
\end{aligned}
$$

Since the normalized spreading codes are used, that is, $\left[s^{u}(l)\right]^{2}=1 / L_{S}$ and $\varepsilon\left\{g_{k i}^{(u)}\left[g_{k i}^{\left(u^{\prime}\right)}\right]\right\}=\sigma_{e}^{2} \delta\left(i-i^{\prime}\right) \delta\left(u-u^{\prime}\right),(10)$ can be written as

$$
\begin{aligned}
\sigma_{f_{k}}^{2}(l)= & \sum_{u=1}^{U} \sum_{i=1}^{n_{T}} \mathcal{\varepsilon}\left\{\left[g_{k i}^{(u)} \sqrt{P_{i}^{(u)}} x_{i}^{(u)}\right]\left[g_{k i}^{(u)} \sqrt{P_{i}^{(u)}} x_{i}^{(u)}\right]^{*}\right\} \\
& \times\left\{s^{u}(l)\left[s^{u}(l)\right]^{*}\right\} \\
= & \frac{1}{L_{S}} \sum_{u=1}^{U} \sum_{i=1}^{n_{T}} P_{i}^{(u)} \varepsilon\left\{g_{k i}^{(u)}\left[g_{k i}^{(u)}\right]^{*}\right\} \mathcal{\varepsilon}\left\{x_{i}^{(u)}\left[x_{i}^{(u)}\right]^{*}\right\} \\
= & \frac{1}{L_{S}} \sum_{u=1}^{U} \sum_{i=1}^{n_{T}} P_{i}^{(u)} \varepsilon\left\{\left|g_{k i}^{(u)}\right|^{2}\right\} \varepsilon\left\{\left|x_{k i}^{(u)}\right|^{2}\right\} \\
= & \frac{\sigma_{e}^{2}}{L_{S}} \sum_{u=1}^{U} \sum_{i=1}^{n_{T}} P_{i}^{(u)}=\frac{U n_{T} \sigma_{e}^{2}}{L_{S}} .
\end{aligned}
$$

Because $\hat{\mathbf{n}}(l)=\mathbf{f}(l)+\mathbf{n}(l)$, the component of $\hat{\mathbf{n}}(l)$ is given as $\hat{n}_{k}(l)=f_{k}(l)+n_{k}(l), k=1, \ldots, n_{R}$ by the aforementioned definition with the variance calculated as

$$
\begin{aligned}
\sigma_{\hat{n}_{k}}^{2}(l) & =\varepsilon\left[\hat{n}_{k}(l) \hat{n}_{k}^{*}(l)\right]=\varepsilon\left\{\left[f_{k}(l)+n_{k}(l)\right]\left[f_{k}(l)+n_{k}(l)\right]^{*}\right\} \\
& =\varepsilon\left\{\left|f_{k}(l)\right|^{2}\right\}+\varepsilon\left\{\left|n_{k}(l)\right|^{2}\right\}=\frac{U n_{T} \sigma_{e}^{2}}{L_{S}}+\sigma_{n}^{2} .
\end{aligned}
$$

Each component of $\widehat{\mathbf{n}}(l)$ is i.i.d zero-mean complex Gaussian variables with the variance of $U n_{T} \sigma_{e}^{2} / L_{S}+\sigma_{n}^{2}$, so the variance of $\hat{\mathbf{n}}(l)$ is $\sigma_{\hat{n}}^{2}(l)=\left(U n_{T} \sigma_{e}^{2} / L_{S}+\sigma_{n}^{2}\right) \mathbf{I}_{n_{R}}$. When $\sigma_{e}^{2}=0$, that is, the channel matrix is estimated perfectly, the variance of the equivalent noise is $\sigma_{\hat{n}}^{2}=\sigma_{n}^{2}$, which is equal to the variance of the scenario where the channel is estimated perfectly.

3.2. Decorrelating Scheme. Optimal coherent and noncoherent detection based on maximum likelihood (ML) processing can be readily derived from $(4)[14,15]$. These optimal detectors perform joint detection for all users and incur an exponential complexity in both the number of active users and that of transmit antennas. The enormous complexity of the optimum receivers renders them mainly of theoretical interest. 
In this part, a suboptimal decorrelating receiver is presented which decouples the detection of different users with a linear complexity in the number of active users [16].

In order to express it compactly, we define

$$
\begin{aligned}
\mathbf{Y} & \triangleq\left[\mathbf{y}(1), \ldots, \mathbf{y}(l), \ldots, \mathbf{y}\left(L_{S}\right)\right], \\
\hat{\mathbf{N}} & \triangleq\left[\widehat{\mathbf{n}}(1), \ldots, \widehat{\mathbf{n}}(l), \ldots, \widehat{\mathbf{n}}\left(L_{S}\right)\right], \\
\mathbf{s}^{(u)} & \triangleq\left[s^{(u)}(1), \ldots, s^{(u)}(l), \ldots, s^{(u)}\left(L_{S}\right)\right], \\
\mathbf{S} & \triangleq\left\{\left[\mathbf{s}^{(1)}\right]^{T}, \ldots,\left[\mathbf{s}^{(u)}\right]^{T}, \ldots,\left[\mathbf{s}^{(U)}\right]^{T}\right\}^{T}, \\
\widehat{\mathbf{A}}^{(u)} & \triangleq \hat{\mathbf{H}}^{(u)} \widehat{\mathbf{V}}^{(u)} \mathbf{P}^{(u)} \mathbf{x}^{(u)}, \\
\widehat{\mathbf{A}} & \triangleq\left[\hat{\mathbf{A}}^{(1)}, \ldots, \hat{\mathbf{A}}^{(u)}, \ldots, \widehat{\mathbf{A}}^{(U)}\right] .
\end{aligned}
$$

Then, (4) is expressed as

$$
\mathbf{Y}=\widehat{\mathbf{A}} \mathbf{S}+\hat{\mathbf{N}} \text {. }
$$

The variance of any component of $\hat{\mathbf{N}}$ is $\sigma_{\hat{n}}^{2}(l)=\left(U n_{T} \sigma_{e}^{2} / L_{S}+\right.$ $\left.\sigma_{n}^{2}\right) \mathbf{I}_{n_{R}}$, so the variance of $\hat{\mathbf{N}}$ is $\sigma_{\hat{N}}^{2}(l)=\varepsilon\left[\hat{\mathbf{N}} \hat{\mathbf{N}}^{H}\right]=$ $\left(U n_{T} \sigma_{e}^{2} / L_{S}+\sigma_{n}^{2}\right) \mathbf{I}_{n_{R}}$ too.

Since the information about all the transmitted symbols is contained in $\hat{\mathbf{A}}$, the key to the differential and coherent receivers introduced in this paper is to obtain an initial estimate of $\widehat{\mathbf{A}}$, which is used for the subsequent detection.

When we assume that $\mathbf{S S}^{H}$ has a full rank, the ML estimate of $\hat{\mathbf{A}}$, which is conditioned on $\left\{\hat{\mathbf{H}}^{(u)}\right\}_{u=1}^{U},\left\{\widehat{\mathbf{V}}^{(u)}\right\}_{u=1}^{U}$, $\mathbf{P}^{(u)}$ and $\left\{\mathbf{S}^{(u)}\right\}_{u=1}^{U}$, is given by

$$
\mathbf{R}=\mathbf{Y} \mathbf{S}^{H}\left(\mathbf{S S}^{H}\right)^{-1}
$$

The equation of (15) is suitable for all the spreading structure, but if the nonorthogonal spreading structures, such as GOLD code, are used, it is very difficult to get the variance of the equivalent noise. We use normalized orthogonal WALSH code in this paper, which satisfies $\mathbf{S S}^{H}=$ $\mathbf{I}_{U}$.

The ML estimate $\mathbf{R}$ is effectively the output of a decorrelator with the input being the received matrix $\mathbf{Y}$. The decorrelator mitigates the MUI and decouples the detection of different users.

Then we study the impact of the decorrelation on the variance of the equivalent noise, via (14), (15) which can be rewritten as

$$
\begin{aligned}
\mathbf{R} & =(\widehat{\mathbf{A}} \mathbf{S}+\hat{\mathbf{N}}) \mathbf{S}^{H}\left(\mathbf{S} \mathbf{S}^{H}\right)^{-1}=\widehat{\mathbf{A}} \mathbf{S} \mathbf{S}^{H}\left(\mathbf{S} \mathbf{S}^{H}\right)^{-1}+\widehat{\mathbf{N}} \mathbf{S}^{H}\left(\mathbf{S} \mathbf{S}^{H}\right)^{-1} \\
& =\widehat{\mathbf{A}}+\widehat{\mathbf{N}} \mathbf{S}^{H}=\widehat{\mathbf{A}}+\widehat{\hat{\mathbf{N}}}
\end{aligned}
$$

where $\hat{\mathbf{N}}=\hat{\mathbf{N}} \mathbf{S}^{H}$ is the equivalent noise after decorrelating and the variance of $\hat{\mathbf{N}}$ is calculated as follows:

$$
\begin{aligned}
\sigma_{\hat{\mathbf{N}}}^{2} & =\varepsilon\left\{\hat{\mathbf{N}} \hat{\hat{N}}^{H}\right\}=\varepsilon\left\{\left[\hat{\mathbf{N}} \mathbf{S}^{H}\right]\left[\hat{\mathbf{N}} \mathbf{S}^{H}\right]^{H}\right\}=\varepsilon\left\{\hat{\mathbf{N}} \mathbf{S}^{H} \mathbf{S} \hat{\mathbf{N}}^{H}\right\} \\
& =\varepsilon\left\{\hat{\mathbf{N}} \hat{\mathbf{N}}^{H}\right\}=\left(\frac{U n_{T} \sigma_{e}^{2}}{L_{S}}+\sigma_{n}^{2}\right) \mathbf{I}_{n_{R}},
\end{aligned}
$$

that is, the variance of equivalent noise is unchanged after decorrelating.

Due to the block structure of $\hat{\mathbf{A}}$, the ML estimate of the desired user denoted by $\mathbf{r}^{(u)}$ can be easily obtained from $\mathbf{R}$, which is the $u$ th column of $\mathbf{R}$

$$
\begin{aligned}
\mathbf{r}^{(u)} & =\mathbf{R}(:, u)=\hat{\mathbf{H}}^{(u)} \hat{\mathbf{V}}^{(u)} \mathbf{P}^{(u)} \mathbf{x}^{(u)}+\hat{\hat{\mathbf{N}}}(:, u) \\
& =\hat{\boldsymbol{\Lambda}}^{(u)} \mathbf{P}^{(u)} \mathbf{x}^{(u)}+\hat{\hat{\mathbf{n}}}^{(u)},
\end{aligned}
$$

where $\hat{\mathbf{n}}^{(u)}=\hat{\hat{\mathbf{N}}}(:, u)$ is the $u$ th column of $\hat{\hat{\mathbf{N}}}$, whose variance is $\sigma_{\hat{\mathbf{n}}(u)}^{2}=\left(U n_{T} \sigma_{e}^{2} / L_{S}+\sigma_{n}^{2}\right) \mathbf{I}_{n_{R}}$, that is, the variance is increased with the number of active users.

The received signal of the desired user is achieved after decorrelating, corresponding to the preprocessing at the transmitter, and the decoupled signal is first orthogonally converted by $\hat{\boldsymbol{\Phi}}^{(u)}$, which is denoted as

$$
\begin{aligned}
\widetilde{\mathbf{r}}^{(u)}= & {\left[\hat{\boldsymbol{\Phi}}^{(u)}\right]^{H} \mathbf{r}^{(u)}=\left[\hat{\boldsymbol{\Phi}}^{(u)}\right]^{H} \hat{\mathbf{H}}^{(u)} \hat{\mathbf{V}}^{(u)} \mathbf{P}^{(u)} \mathbf{x}^{(u)} } \\
& +\left[\hat{\boldsymbol{\Phi}}^{(u)}\right]^{H} \hat{\mathbf{n}}^{(u)}=\hat{\Lambda}^{(u)} \mathbf{P}^{(u)} \mathbf{x}^{(u)}+\hat{\hat{\mathbf{n}}}^{(u)}
\end{aligned}
$$

where $\hat{\hat{\mathbf{n}}}^{(u)}=\left[\hat{\boldsymbol{\Phi}}^{(u)}\right]^{H} \widehat{\hat{\mathbf{n}}}^{(u)}$ the variance of the equivalent noise $\hat{\hat{\mathbf{n}}}^{(u)}$ is calculated as follows:

$$
\begin{aligned}
& \varepsilon\left\{\hat{\hat{\mathbf{n}}}^{(u)}(l)\right\}=\varepsilon\left\{\left[\hat{\boldsymbol{\Phi}}^{(u)}\right]^{H} \hat{\hat{\mathbf{n}}}^{(u)}\right\} \\
& =\left[\hat{\boldsymbol{\Phi}}^{(u)}\right]^{H} \varepsilon\left\{\hat{\mathbf{n}}^{(u)}\right\}=\mathbf{0}_{n_{R}}, \\
& \varepsilon\left\{\left[\hat{\hat{\mathbf{n}}}^{(u)}(l)\right]\left[\hat{\hat{\mathbf{n}}}^{(u)}(l)\right]^{H}\right\}=\varepsilon\left\{\left\{\left[\hat{\boldsymbol{\Phi}}^{(u)}\right]^{H} \hat{\hat{\mathbf{n}}}^{(u)}\right\}\right. \\
& \left.\times\left\{\left[\widehat{\boldsymbol{\Phi}}^{(u)}\right]^{H} \widehat{\hat{\mathbf{n}}}^{(u)}\right\}^{H}\right\} \\
& =\varepsilon\left\{\left[\hat{\Phi}^{(u)}\right]^{H} \hat{\hat{\mathbf{n}}}^{(u)}\left[\hat{\hat{\mathbf{n}}}^{(u)}\right]^{H} \hat{\boldsymbol{\Phi}}^{(u)}\right\} \\
& =\left[\hat{\boldsymbol{\Phi}}^{(u)}\right]^{H} \varepsilon\left\{\hat{\hat{\mathbf{n}}}^{(u)}\left[\hat{\hat{\mathbf{n}}}^{(u)}\right]^{H}\right\} \hat{\boldsymbol{\Phi}}^{(u)} \\
& =\left(\frac{U n_{T} \sigma_{e}^{2}}{L_{S}}+\sigma_{n}^{2}\right) \mathbf{I}_{n_{R}} \text {. }
\end{aligned}
$$


Clearly, the components of $\hat{\hat{\mathbf{n}}}^{(u)}$ are also i.i.d zero-mean Gaussian variables with the variance of $\left(U n_{T} \sigma_{e}^{2} / L_{S}+\sigma_{n}^{2}\right)$.

3.3. SVD-Aided Beamforming and Power Allocation Scheme. Given the proposed equivalent multiuser Turbo-BLAST system model, we give an SVD-aided beamforming and transmit power allocation scheme in this part, which aims at maximizing the capacity performance of the desired user in the presence of imperfect CSI.

When CSI is imperfect, the right singular matrix of $\hat{\mathbf{H}}^{(u)}$ is used for beamforming and the channel capacity of the desired user with SVD-aided beamforming and power allocation can be expressed as $[1,18]$

$$
\begin{array}{r}
C^{(u)}\left[\overline{\hat{\lambda}}^{(u)}\right]=\sum_{k=1}^{n_{T}} \log _{2}\left[1+\frac{P_{k}^{(u)} \hat{\lambda}_{k}^{(u)}}{\left(U n_{T} \sigma_{e}^{2}\right) / L_{S}+\sigma_{n}^{2}}\right], \\
u=1, \ldots, U,
\end{array}
$$

where $\overline{\hat{\lambda}}^{(u)}=\operatorname{diag}\left[\hat{\lambda}_{1}^{(u)}, \ldots, \hat{\lambda}_{n_{T}}^{(u)}\right]$ is the diagonal matrix with the eigenvalues of $\hat{\mathbf{H}}^{(u)}\left[\hat{\mathbf{H}}^{(u)}\right]^{H}$ and $P_{k}^{(u)}$ is the transmit power allocated for the $k$ th subchannel of the desired user, which satisfies the total transmit power constrain. The capacity subject to an optimization problem is given as below

$$
\begin{aligned}
& {\left[P_{1}^{(u)}, \ldots, P_{n_{T}}^{(u)}\right]_{\mathrm{opt}}} \\
& =\arg \max \left\{\sum_{k=1}^{n_{T}} \log _{2}\left[1+\frac{P_{k}^{(u)} \hat{\lambda}_{k}^{(u)}}{\left(U n_{T} \sigma_{e}^{2}\right) / L_{S}+\sigma_{n}^{2}}\right]\right\} \\
& \quad \text { subject to } \sum_{k=1}^{n_{T}} P_{k}^{(u)}=P_{\text {Total. }}
\end{aligned}
$$

The Lagrange multiplier method is employed to find the optimum power allocation matrix that can maximize the capacity with total power constraint. After solving the equations in (23), we find that the optimal power allocated for the $k$ th subchannel is

$$
P_{k}^{(u)}=\left\{\mu-\left(\frac{U n_{T} \sigma_{e}^{2}}{L_{S}}+\sigma_{n}^{2}\right)\left[\hat{\lambda}_{k}^{(u)}\right]^{-1}\right\}^{+}
$$

where $(x)^{+} \triangleq \max \{x, 0\}$ and $\mu$ is the Lagrange multiplier restricted by $\sum_{k=1}^{n_{T}}\left\{\mu-\left(U n_{T} \sigma_{e}^{2} / L_{S}+\sigma_{n}^{2}\right)\left[\hat{\lambda}_{k}^{(u)}\right]^{-1}\right\}^{+}=P_{\text {Total }}$.
In a frequency-nonselective, Rayleigh fading MIMO channel, the channel capacity is the statistical mean of $\overline{\hat{\lambda}}^{(u)}$ approximately in the presence of CSI imperfection.

$$
\begin{aligned}
C^{(u)} & =\varepsilon \overline{\hat{\lambda}}\left[C^{(u)}\left(\overline{\hat{\lambda}}^{(u)}\right)\right] \\
& =\varepsilon\left\{\sum_{k=1}^{n_{T}} \log _{2}\left[1+\frac{\mathbf{P}_{k}^{(u)} \hat{\lambda}_{k}^{(u)}}{\left(U n_{T} \sigma_{e}^{2}\right) / L_{S}+\sigma_{n}^{2}}\right]\right\} \\
& =\varepsilon\left\{\sum_{k=1}^{n_{T}}\left[\log _{2}\left(\mu \hat{\lambda}_{k}^{(u)}\right)\right]^{+}\right\} .
\end{aligned}
$$

Because $\left\{\hat{\lambda}_{k}^{(u)}\right\}_{k=1}^{n_{T}}$ are i.i.d variables and all of them have the same probability density $p\left[\hat{\lambda}_{k}^{(u)}\right]$, formula $(25)$ can be expressed as

$$
C^{(u)}=n_{T} \int_{0}^{+\infty}\left[\log _{2}\left(\mu \hat{\lambda}_{k}^{(u)}\right)\right] p\left(\hat{\lambda}_{k}^{(u)}\right) d \hat{\lambda}_{k}^{(u)}, \quad u=1, \ldots, U .
$$

Since singular values are random variables, it is hard to achieve the function of probability density straightly. The channel capacity can be calculated by averaging (26) over a large number of channel realizations, which is expressed as

$$
\begin{aligned}
C^{(u)} & \approx \varepsilon\left\{\sum_{k=1}^{n_{T}} \log _{2}\left[1+\frac{\mathbf{P}_{k}^{(u)} \hat{\lambda}_{k}^{(u)}}{\left(U n_{T} \sigma_{e}^{2}\right) / L_{S}+\sigma_{n}^{2}}\right]\right\} \\
& \approx \frac{1}{N} \sum_{i=1}^{N}\left\{\sum_{k=1}^{n_{T}} \log _{2}\left[1+\frac{P_{k, i}^{(u)} \hat{\lambda}_{k, i}^{(u)}}{\left(U n_{T} \sigma_{e}^{2}\right) / L_{S}+\sigma_{n, i}^{2}}\right]\right\}, \\
u & =1, \ldots, U,
\end{aligned}
$$

where $N$ is the number of channel realizations, which is used for simulation, $\left\{P_{k, i}^{(u)}\right\}_{k=1}^{n_{T}}$ are the transmit power sequence for ith channel matrix $\hat{\mathbf{H}}_{i}^{(u)},\left\{\hat{\lambda}_{k, i}^{(u)}\right\}_{k=1}^{n_{T}}$ are eigenvalues of $\hat{\mathbf{H}}_{i}^{(u)}\left[\hat{\mathbf{H}}_{i}^{(u)}\right]^{H}$, and $\sigma_{e, i}^{2}$ and $\sigma_{n, i}^{2}$ are the variances of channel estimation error and AWGN, respectively.

3.4. Iterative Detection Algorithm for the Uplink of Multiuser Turbo-BLAST Systems. In this section, an iterative detection strategy is employed to enhance the error performance [4] in the presence of imperfect CSI. Because the orthonormal spreading codes are used, the MUI is removed absolutely after decorrelating, so the iterative detection of every user is completely independent from each other.

Let $x_{k}^{(u)}$ be the $k$ th transmitted signal of desired user at a sampling epoch, by (19); the received symbol vector $\widetilde{\mathbf{r}}^{(u)}$ which is corrupted by the channel noise and interferences, can be written as the sum of desired response, the CAI, and the equivalent noise:

$$
\tilde{\mathbf{r}}^{(u)}=\hat{\boldsymbol{\lambda}}_{k}^{(u)} \sqrt{P_{k}^{(u)}} x_{k}^{(u)}+\hat{\Lambda}_{\bar{k}}^{(u)} \mathbf{P}_{\bar{k}}^{(u)} \mathbf{x}_{\bar{k}}^{(u)}+\hat{\hat{\mathbf{n}}}^{(u)} .
$$


In (27), $\hat{\lambda}_{k}^{(u)}$ is the $k$ th column of the matrix $\hat{\Lambda}^{(u)}, P_{k}^{(u)}$ is the transmit power allocated to the $k$ th subchannel of the desired user, and $\hat{\Lambda}_{\bar{k}}^{(u)}, \mathbf{x}_{\bar{k}}^{(u)}, \mathbf{P}_{\bar{k}}^{(u)}$ is defined as follows:

$$
\begin{aligned}
& \hat{\Lambda}_{\bar{k}}^{(u)}=\left[\hat{\lambda}_{1}^{(u)}, \ldots, \hat{\lambda}_{k-1}^{(u)}, \hat{\lambda}_{k+1}^{(u)}, \ldots, \hat{\lambda}_{n_{T}}^{(u)}\right], \\
& \mathbf{x}_{\bar{k}}^{(u)}=\left[x_{1}^{(u)}, \ldots, x_{k-1}^{(u)}, x_{k+1}^{(u)}, \ldots, x_{n_{T}}^{(u)}\right]^{T}, \\
& \mathbf{P}_{\bar{k}}^{(u)}=\operatorname{diag}\left(\sqrt{P_{1}^{(u)}}, \ldots, \sqrt{P_{k-1}^{(u)}}, \sqrt{P_{k+1}^{(u)}}, \ldots, \sqrt{P_{n_{T}}^{(u)}}\right) .
\end{aligned}
$$

By (29), the decision statistic of $k$ th substream of the $u$ th user using a linear filter $\widehat{\mathbf{w}}_{k}^{(u)}$ can be expressed as

$$
\begin{aligned}
y_{k}^{(u)}= & \underbrace{\left[\hat{\mathbf{w}}_{k}^{(u)}\right]^{H} \hat{\boldsymbol{\lambda}}_{k}^{(u)} \sqrt{P_{k}^{(u)}} x_{k}^{(u)}}_{\hat{q}_{k}^{(u)}} \\
& +\underbrace{\left[\hat{\mathbf{w}}_{k}^{(u)}\right]^{H} \hat{\boldsymbol{\Lambda}}_{\bar{k}}^{(u)} \mathbf{P}_{\bar{k}}^{(u)} \mathbf{x}_{\bar{k}}^{(u)}}_{\hat{d}_{k}^{(u)}}+\underbrace{\left[\hat{\mathbf{w}}_{k}^{(u)}\right]^{H} \hat{\hat{\mathbf{n}}}^{(u)}}_{\hat{z}_{k}^{(u)}},
\end{aligned}
$$

where $\hat{q}_{k}^{(u)}, \hat{d}_{k}^{(u)}$, and $\hat{z}_{k}^{(u)}$ are the desired response obtained by the linear filter, the coantenna interference, and the phaserotated equivalent noise, respectively.

The CAI can be removed from $y_{k}^{(u)}$ by the proposed iterative detector and soft interference cancellation based on mean square error (MMSE) principle. The improved estimation of the transmitted symbol $\widetilde{x}_{k}^{(u)}$ can be formulated as

$$
\tilde{x}_{k}^{(u)}=\left[\widehat{\mathbf{w}}_{k}^{(u)}\right]^{H} \widetilde{\mathbf{r}}^{(u)}-\widehat{d}_{k}^{(u)},
$$

where $\tilde{x}_{k}^{(u)}$ is the estimate of the symbol $x_{k}^{(u)}$ and $\hat{d}_{k}^{(u)}$ is the linear combination of the interfering substreams. The estimation error is defined as $\Delta x_{k}^{(u)}=\tilde{x}_{k}^{(u)}-x_{k}^{(u)}$. The weighted vector $\widehat{\mathbf{w}}_{k}^{(u)}$ and the interference combination $\hat{d}_{k}^{(u)}$ are optimized by minimizing the mean-square estimation error $\Delta x_{k}^{(u)}$ between each substream and the related estimation, by the following cost function:

$$
\left[\hat{\mathbf{w}}_{k}^{(u)}, \hat{d}_{k}^{(u)}\right]=\operatorname{argmin} \varepsilon\left[\left\|\tilde{x}_{k}^{(u)}-x_{k}^{(u)}\right\|^{2}\right]
$$

where the expectation is taken over the equivalent noise $\hat{\hat{\mathbf{n}}}$ and the statistics of the data sequence $\mathbf{x}^{(u)}$.
We use standard minimization techniques to solve the optimization problem formulated in (32). In order to arrive at the solution, we write the cost function as

$$
\begin{aligned}
\operatorname{Cost}^{(u)}= & \varepsilon\left[\left\|\tilde{x}_{k}^{(u)}-x_{k}^{(u)}\right\|^{2}\right] \\
= & \varepsilon\left[\left\|\left(\hat{\mathbf{w}}_{k}^{(u)}\right)^{H} \widetilde{\mathbf{r}}^{(u)}-\hat{d}_{k}^{(u)}-x_{k}^{(u)}\right\|^{2}\right] \\
= & {\left[\widehat{\mathbf{w}}_{k}^{(u)}\right]^{H} \varepsilon\left\{\left\{\tilde{\mathbf{r}}^{(u)}\left[\widetilde{\mathbf{r}}^{(u)}\right]^{H}\right\} \hat{\mathbf{w}}_{k}^{(u)}\right.} \\
& -\left[\widehat{\mathbf{w}}_{k}^{(u)}\right]^{H} \varepsilon\left\{\tilde{\mathbf{r}}^{(u)}\left[\hat{d}_{k}^{(u)}+x_{k}^{(u)}\right]^{*}\right\} \\
& -\varepsilon\left\{\tilde{\mathbf{r}}^{(u)}\left[\hat{d}_{k}^{(u)}+x_{k}^{(u)}\right]^{*}\right\}^{H} \hat{\mathbf{w}}_{k}^{(u)} \\
& +\varepsilon\left\{\left[\hat{d}_{k}^{(u)}+x_{k}^{(u)}\right]\left[\hat{d}_{k}^{(u)}+x_{k}^{(u)}\right]^{*}\right\},
\end{aligned}
$$

where

$$
\begin{aligned}
& \varepsilon\left\{\widetilde{\mathbf{r}}^{(u)}\left[\widetilde{\mathbf{r}}^{(u)}\right]^{H}\right\}=\varepsilon\left\{\left[\hat{\boldsymbol{\lambda}}_{k}^{(u)} \sqrt{P_{k}^{(u)}} x_{k}^{(u)}\right.\right. \\
& \left.+\widehat{\Lambda}_{\bar{k}}^{(u)} \mathbf{P}_{\bar{k}}^{(u)} \mathbf{x}_{\bar{k}}^{(u)}+\hat{\hat{\mathbf{n}}}^{(u)}\right] \\
& \times\left[\hat{\lambda}_{k}^{(u)} \sqrt{P_{k}^{(u)}} x_{k}^{(u)}\right. \\
& \left.\left.+\hat{\boldsymbol{\Lambda}}_{\bar{k}}^{(u)} \mathbf{P}_{\bar{k}}^{(u)} \mathbf{x}_{\bar{k}}^{(u)}+\hat{\hat{\mathbf{n}}}^{(u)}\right]^{H}\right\} \\
& =\left[\hat{\lambda}_{k}^{(u)} \sqrt{P_{k}^{(u)}}\right]\left[\hat{\lambda}_{k}^{(u)} \sqrt{P_{k}^{(u)}}\right]^{H} \\
& +\left[\hat{\Lambda}_{\bar{k}}^{(u)} \mathbf{P}_{\bar{k}}^{(u)}\right] \mathcal{\varepsilon}\left\{\mathbf{x}_{\bar{k}}^{(u)}\left[\mathbf{x}_{\bar{k}}^{(u)}\right]^{H}\right\} \\
& \times\left[\hat{\Lambda}_{\bar{k}}^{(u)} \mathbf{P}_{\bar{k}}^{(u)}\right]^{H} \\
& +\left(\frac{U n_{T} \sigma_{e}^{2}}{L_{S}}+\sigma_{n}^{2}\right) \mathbf{I}_{n_{R}} \\
& \varepsilon\left\{\widetilde{\mathbf{r}}^{(u)}\left[\hat{d}_{k}^{(u)}+x_{k}^{(u)}\right]^{*}\right\}=\varepsilon\left\{\left[\hat{\lambda}_{k}^{(u)} \sqrt{\mathbf{P}_{k}^{(u)}} \mathbf{x}_{k}^{(u)}\right.\right. \\
& \left.+\widehat{\Lambda}_{\bar{k}}^{(u)} \mathbf{P}_{\bar{k}}^{(u)} \mathbf{x}_{\bar{k}}^{(u)}+\hat{\hat{\mathbf{n}}}^{(u)}\right] \\
& \left.\times\left[\hat{d}_{k}^{(u)}+x_{k}^{(u)}\right]^{*}\right\} \\
& =\hat{\lambda}_{k}^{(u)} \sqrt{\mathbf{P}_{k}^{(u)}} \\
& +\hat{\Lambda}_{\bar{k}}^{(u)} \mathbf{P}_{\bar{k}}^{(u)} \varepsilon\left[\mathbf{x}_{\bar{k}}^{(u)}\right]\left[\hat{d}_{\bar{k}}^{(u)}\right]^{*} .
\end{aligned}
$$


Via (34), the cost function in (33) can be further evaluated as

$$
\begin{aligned}
\operatorname{Cost}^{(u)}=\left[\hat{\mathbf{w}}_{k}^{(u)}\right]^{H}\{[ & \left.\hat{\lambda}_{k}^{(u)} \sqrt{P_{k}^{(u)}}\right]\left[\hat{\lambda}_{k}^{(u)} \sqrt{P_{k}^{(u)}}\right]^{H} \\
& +\left[\hat{\boldsymbol{\Lambda}}_{\bar{k}}^{(u)} \mathbf{P}_{\bar{k}}^{(u)}\right] \varepsilon\left\{\mathbf{x}_{\bar{k}}^{(u)}\left[\mathbf{x}_{\bar{k}}^{(u)}\right]^{H}\right\}\left[\hat{\boldsymbol{\Lambda}}_{\bar{k}}^{(u)} \mathbf{P}_{\bar{k}}^{(u)}\right]^{H} \\
& \left.+\left(\frac{U n_{T} \sigma_{e}^{2}}{L_{S}}+\sigma_{n}^{2}\right) \mathbf{I}_{n_{R}}\right\} \hat{\mathbf{w}}_{k}^{(u)} \\
& -\left[\hat{\mathbf{w}}_{k}^{(u)}\right]^{H}\left\{\hat{\boldsymbol{\lambda}}_{k}^{(u)} \sqrt{P_{k}^{(u)}}+\hat{\boldsymbol{\Lambda}}_{\bar{k}}^{(u)} \mathbf{P}_{\bar{k}}^{(u)} \varepsilon\left[\mathbf{x}_{k}^{(u)}\right]\left[\hat{d}_{k}^{(u)}\right]^{*}\right\} \\
& -\left\{\hat{\lambda}_{k}^{(u)} \sqrt{P_{k}^{(u)}}+\hat{\boldsymbol{\Lambda}}_{\bar{k}}^{(u)} \mathbf{P}_{\bar{k}}^{(u)} \varepsilon\left\{\mathbf{x}_{k}^{(u)}\right\}\left[\hat{d}_{k}^{(u)}\right]^{*}\right\} \hat{\mathbf{w}}_{k}^{(u)} \\
& +\varepsilon\left\{\hat{d}_{k}^{(u)}\left[\hat{d}_{k}^{(u)}\right]^{*}+x_{k}^{(u)}\left[\hat{d}_{k}^{(u)}\right]^{*}\right. \\
& \left.+\hat{d}_{k}^{(u)}\left[x_{k}^{(u)}\right]^{*}+x_{k}^{(u)}\left[x_{k}^{(u)}\right]^{*}\right\} .
\end{aligned}
$$

The linear combination of interfering substreams $\hat{d}_{k}^{(u)}$ and the weighted vector $\widehat{\mathbf{w}}_{k}^{(u)}$ is obtained in (36) by letting $\partial \operatorname{Cost}^{(u)} / \partial\left[\hat{d}_{k}^{(u)}\right]^{*}=0$ and $\partial \operatorname{Cost}^{(u)} / \partial\left[\hat{\mathbf{w}}_{k}^{(u)}\right]^{*}=0$, respectively,

$$
\begin{aligned}
& \hat{d}_{k}^{(u)}=\left[\hat{\mathbf{w}}_{k}^{(u)}\right]^{H}\left[\hat{\lambda}_{k}^{(u)} \sqrt{P_{k}^{(u)}}\right]\left[\hat{\lambda}_{k}^{(u)} \sqrt{P_{k}^{(u)}}\right]^{H} \varepsilon\left[\mathbf{x}_{\bar{k}}^{(u)}\right], \\
& \hat{\mathbf{w}}_{k}^{(u)}=\left[\mathbf{Q}^{(u)}+\mathbf{S}^{(u)}+\left(\frac{U n_{T} \sigma_{e}^{2}}{L_{S}}+\sigma_{n}^{2}\right) \mathbf{I}_{n_{R}}\right]^{-1}\left[\hat{\lambda}_{k}^{(u)} \sqrt{P_{k}^{(u)}}\right],
\end{aligned}
$$

where

$$
\begin{aligned}
\mathbf{Q}^{(u)}= & {\left[\hat{\boldsymbol{\lambda}}_{k}^{(u)} \sqrt{P_{k}^{(u)}}\right]\left[\hat{\boldsymbol{\lambda}}_{k}^{(u)} \sqrt{P_{k}^{(u)}}\right]^{H}, } \\
\mathbf{S}^{(u)}= & {\left[\hat{\Lambda}_{\bar{k}}^{(u)} \mathbf{P}_{\bar{k}}^{(u)}\right]\left\{\mathbf{I}_{\left(n_{T}-1\right)}-\operatorname{diag}\left\{\varepsilon\left[\mathbf{x}_{\bar{k}}^{(u)}\right] \varepsilon\left[\mathbf{x}_{\bar{k}}^{(u)}\right]^{H}\right\}\right\} } \\
& \times\left[\hat{\Lambda}_{\bar{k}}^{(u)} \mathbf{P}_{\bar{k}}^{(u)}\right]^{H} .
\end{aligned}
$$

Therefore, for the desired user, the weighted vector $\widehat{\mathbf{w}}_{k}^{(u)}$ is used for the iterative detection in the presence of imperfect CSI. Thus (31) can be rewritten as

$$
\begin{aligned}
\widetilde{\mathbf{x}}_{k}^{(u)}= & {\left[\hat{\mathbf{w}}_{k}^{(u)}\right]^{H} \widetilde{\mathbf{r}}^{(u)}-\widehat{d}_{k}^{(u)} } \\
= & {\left[\hat{\lambda}_{k}^{(u)} \sqrt{P_{k}^{(u)}}\right]^{H} } \\
& \times\left\{\left[\mathbf{Q}^{(u)}+\mathbf{S}^{(u)}+\left(\frac{U n_{T} \sigma_{e}^{2}}{L_{S}}+\sigma_{n}^{2}\right) \mathbf{I}_{n_{R}}\right]^{-1}\right\} \\
& \times\left\{\mathbf{r}^{(u)}-\left[\hat{\Lambda}_{\bar{k}}^{(u)} \mathbf{P}_{\bar{k}}^{(u)}\right] \varepsilon\left[\mathbf{x}_{\bar{k}}^{(u)}\right]\right\} .
\end{aligned}
$$

For the first iteration, we assume $\varepsilon\left[\mathbf{x}_{\bar{k}}^{(u)}\right]=0$ and thus the linear MMSE detection for the $k$ th substream of the desired user becomes

$$
\begin{aligned}
\tilde{x}_{k}^{(u)}= & {\left[\hat{\lambda}_{k}^{(u)} \sqrt{P_{k}^{(u)}}\right]^{H} } \\
& \times\left\{\left[\mathbf{Q}^{(u)}+\mathbf{S}^{(u)}+\left(\frac{U n_{T} \sigma_{e}^{2}}{L_{S}}+\sigma_{n}^{2}\right) \mathbf{I}_{n_{R}}\right]^{-1}\right\} \tilde{\mathbf{r}}^{(u)} .
\end{aligned}
$$

Next, we assume that $\varepsilon\left[\mathbf{x}_{\bar{k}}^{(u)}\right] \rightarrow \mathbf{x}_{\bar{k}}^{(u)}$ with the increasing number of iterations, the CAI interference canceller for the $k$ th substream can be reduced to the linear MMSE receiver expressed as (40), where the SVD-assisted beamforming and power allocation scheme are used

$$
\begin{aligned}
\tilde{x}_{k}^{(u)}= & {\left[\hat{\boldsymbol{\lambda}}_{k}^{(u)} \sqrt{P_{k}^{(u)}}\right]^{H} } \\
& \times\left\{\left[\mathbf{Q}^{(u)}+\mathbf{S}^{(u)}+\left(\frac{U n_{T} \sigma_{e}^{2}}{L_{S}}+\sigma_{n}^{2}\right) \mathbf{I}_{n_{R}}\right]^{-1}\right\} \\
& \times\left\{\tilde{\mathbf{r}}^{(u)}-\left[\hat{\boldsymbol{\Lambda}}_{\bar{k}}^{(u)} \mathbf{P}_{\bar{k}}^{(u)}\right] \mathbf{x}_{\bar{k}}^{(u)}\right\} .
\end{aligned}
$$

The performance of iterative scheme depends on the veracity of channel estimation matrix and the power allocation scheme

\section{Simulation Results}

In this section, we compare the capacity and BER performance of the traditional and modified multiuser TurboBLAST systems, where the traditional multiuser TurboBLAST systems adopt the equal power allocated strategy and without the SVD-aided preprocessing and postprocessing, denoted by "EPA", and the modified multiuser Turbo-BLAST systems employ the proposed SVD-aided beamforming and transmit power allocation scheme, denoted by "BF-TPA", respectively.

At the transmitter, the data stream is first encoded by a rate- $1 / 2$ convolutional code with generator $(7,5)$, modulated by 8 -PSK modulation scheme, and spread by WALSH codes, whose capacity performance over a large number of channel realizations is exhibited in Figures 3-5, extra capacity, which is defined as the capacity gap between the modified multiuser Turbo-BLAST system and the conventional one, is given in Figure 6, and finally, BER performance is presented in Figures 7-8 with different inaccuracy of channel estimation and different number of active users. 4 active users are considered in Figure 3 and Figures 5-7.

Figure 3 shows capacity performance of traditional and modified multiuser Turbo-BLAST systems with four transmit and four receive antennas under the conditions of different channel estimation errors. Figure 3 indicates that the proposed SVD-aided beamforming and transmit power allocation strategy is an effective means to improve capacity performance even with the imperfect CSI, and the larger 


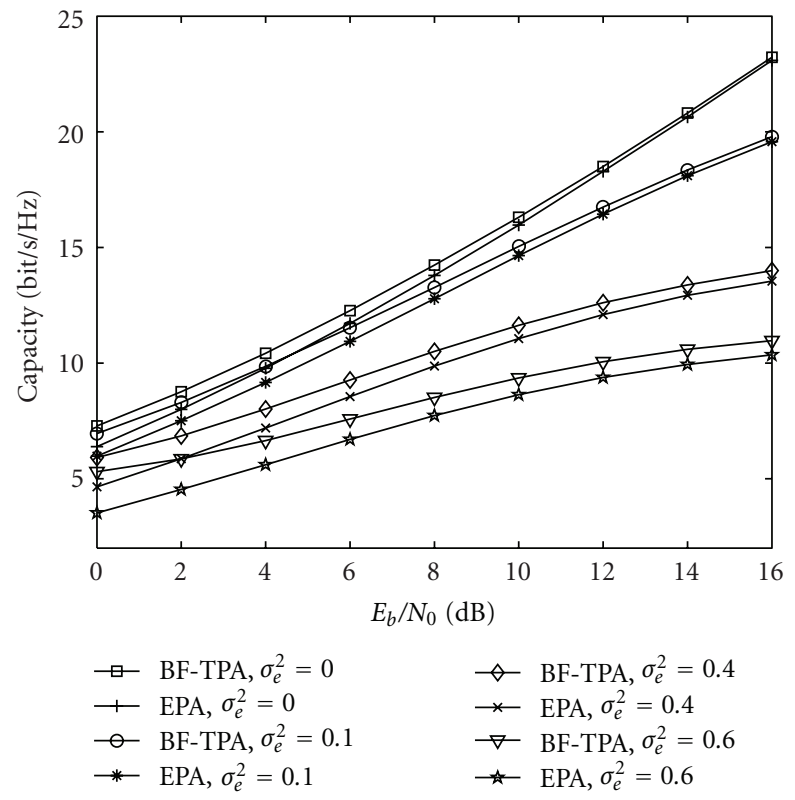

FIgURE 3: Capacity performance of traditional and modified multiuser Turbo-BLAST systems $(U=4)$.

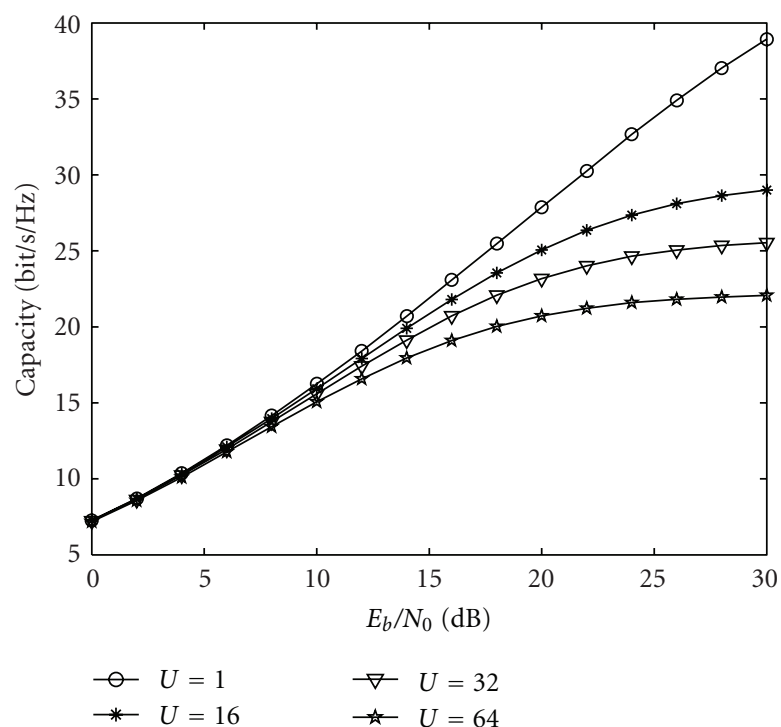

FIGURE 4: Capacity performance of the modified multiuser Turbo-BLAST systems with different number of active users in the presence of imperfect CSI $\left(\sigma_{e}^{2}=0.01\right)$.

capacity gains can be achieved for lower $E_{b} / N_{0}$ and/or higher $\sigma_{e}^{2}$. For example, at $E_{b} / N_{0}=10 \mathrm{~dB}$, there are $0.4(\mathrm{bit} / \mathrm{s} / \mathrm{Hz})$ and $0.8(\mathrm{bit} / \mathrm{s} / \mathrm{Hz})$ capacity gains for $\sigma_{e}^{2}=0.1$ and $\sigma_{e}^{2}=0.4$, respectively.

The capacity performance of the modified multiuser Turbo-BLAST systems with different number of active users in the presence of imperfect CSI are given in Figure 4, where $U$ denotes the number of active users. It is clear that from Figure 4, in the presence of imperfect CSI, the capacity is lower for larger number of active users, and that the impact of the number of active users on the capacity performance is higher for higher $E_{b} / N_{0}$. For instance, at $E_{b} / N_{0}=0 \mathrm{~dB}$, the capacity of desired user is almost the same for different number of active users. As $E_{b} / N_{0}$ becomes high, the capacity degradation becomes large with the number of active users; for example, at $E_{b} / N_{0}=30 \mathrm{~dB}$, there are $17(\mathrm{bit} / \mathrm{s} / \mathrm{Hz})$ capacity degradation for the desired user when system with 64 active users is compared to that with only one active user. This is due to the existence of the channel estimation error, which will make the equivalent noise of the system large, especially for large $U$ (which can be seen from (17) and (21)). This large noise will degrade the system performance greatly because it can affect 


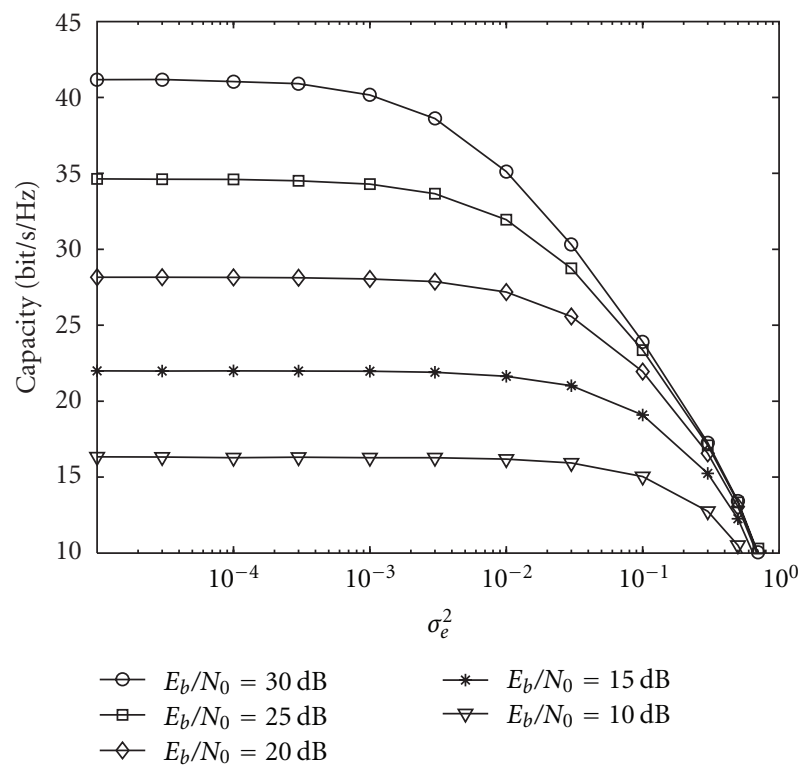

Figure 5: Capacity performance of the modified multiuser Turbo-BLAST system in different values of $E_{b} / N_{0}$ with imperfect CSI $(U=4)$.

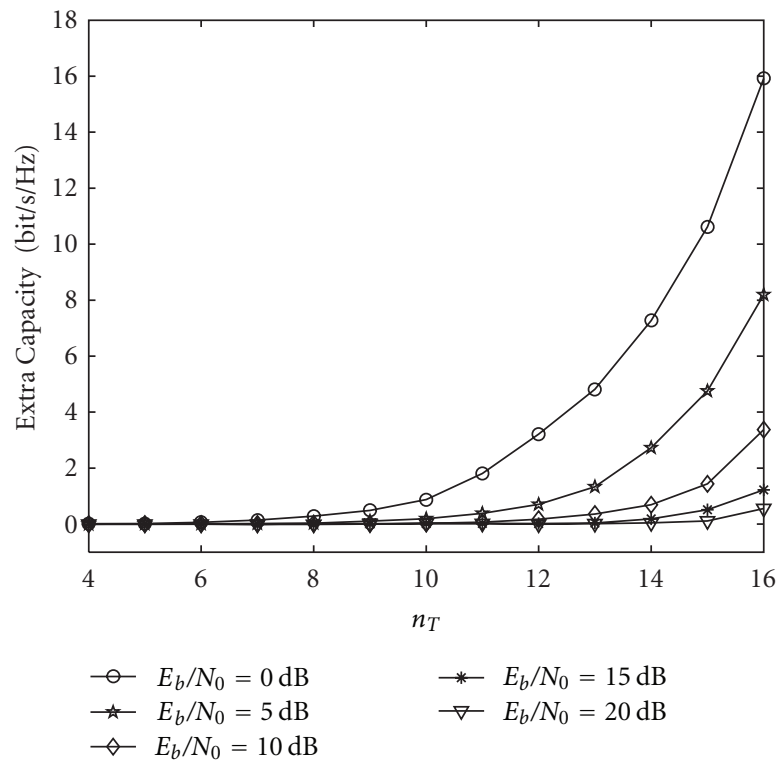

FIGURE 6: Extra capacity results between modified and traditional multiuser Turbo-BLAST systems with sixteen receive antennas and various transmit antennas configuration under the conditions of imperfect CSI $\left(\sigma_{e}^{2}=0.01, U=4\right)$.

the following iterative detection and the resultant capacity calculation.

Figure 5 shows the capacity performance of the modified multiuser Turbo-BLAST systems in different values of $E_{b} / N_{0}$ with imperfect CSI. The simulation results are obtained for five different $E_{b} / N_{0}$ values of $10 \mathrm{~dB}, 15 \mathrm{~dB}, 20 \mathrm{~dB}, 25 \mathrm{~dB}$, and $30 \mathrm{~dB}$, and under various degrees of CSI imperfection. From Figure 5, we see that for CSI imperfection with $\sigma_{e}^{2}<$ $10^{-3}$, the actual capacity is almost constant, as the CSI imperfection gets more severe; in particular, $\sigma_{e}^{2}>10^{-2}$; the capacity becomes very sensitive to the CSI imperfection.
And we see that the CSI imperfection on the capacity performance is higher for higher $E_{b} / N_{0}$ because for higher $E_{b} / N_{0}$, that is, $\sigma_{n}^{2} \rightarrow 0$, the variance of the equivalent noise mainly depends on the error of channel estimation, and therefore, the capacity performance becomes sensitive to the CSI imperfection.

In Figure 6, we give the extra capacity results, which are defined as the capacity gap between modified and traditional multiuser Turbo-BLAST systems with sixteen receive antennas and various transmit antennas configuration under the conditions of imperfect CSI $\left(\sigma_{e}^{2}=0.01\right)$ for five different 


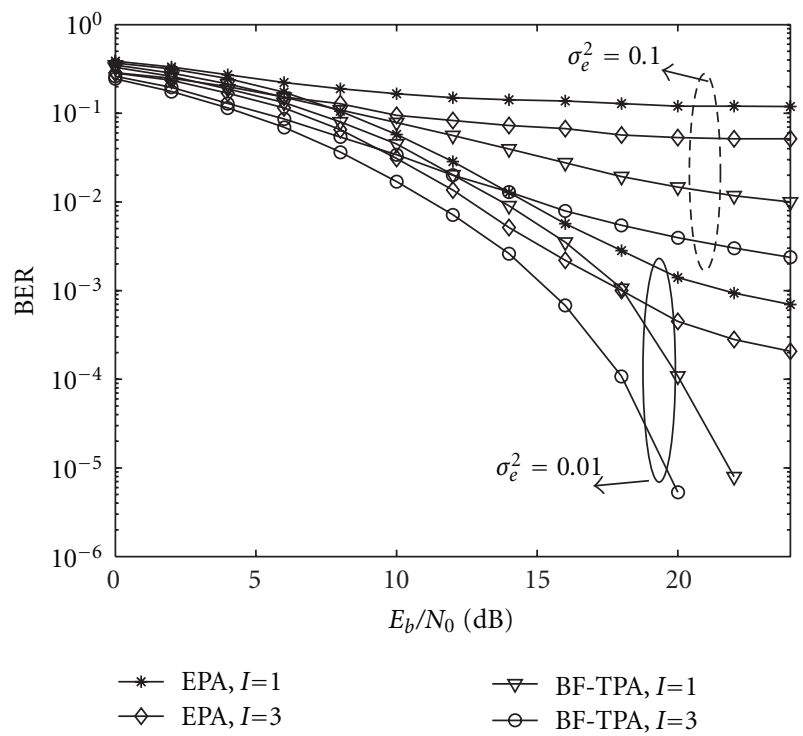

FIGURE 7: BER performance of traditional and modified Turbo-BLAST systems under the conditions of different inaccuracy of channel estimation $(U=4)$.

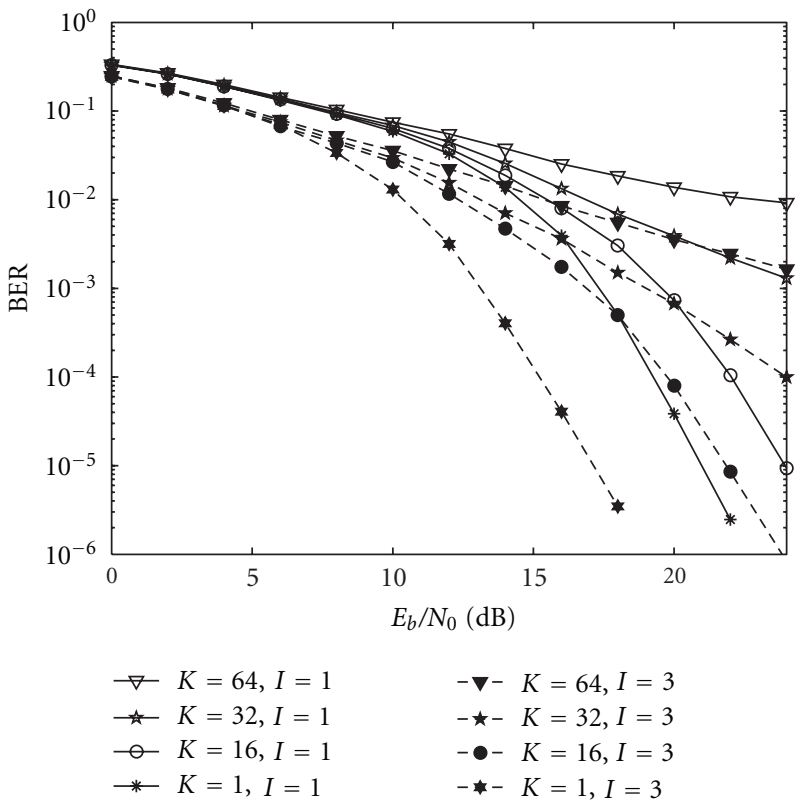

FIGURE 8: BER performance of modified Turbo-BLAST systems with different number of active users under the conditions of imperfect CSI $\left(\sigma_{e}^{2}=0.01\right)$.

$E_{b} / N_{0}$ values of $0 \mathrm{~dB}, 5 \mathrm{~dB}, 10 \mathrm{~dB}, 15 \mathrm{~dB}$, and $20 \mathrm{~dB}$. It is clear that from Figure 6, we can see the extra capacity is higher for lower $E_{b} / N_{0}$ and/or large number of transmit antennas in the presence of CSI imperfection. For instance, at $E_{b} / N_{0}=$ $0 \mathrm{~dB}$, there are $16(\mathrm{bit} / \mathrm{s} / \mathrm{Hz})$ capacity gains for the multiuser Turbo-BLAST system with sixteen transmit antennas, while only $3(\mathrm{bit} / \mathrm{s} / \mathrm{Hz})$ capacity gains can be achieved for twelve transmit antennas.

BER performance of traditional and modified multiuser Turbo-BLAST systems under the condition of different inaccuracy of channel estimation is shown in Figure 7, where
$I$ denotes the number of iterative detection. It is observed from Figure 7 that as the BER results are concerned, the modified multiuser Turbo-BLAST system outperforms the traditional one under the same conditions, regardless of the status of CSI. This implies that the proposed SVDaided beamforming and power allocation strategy are also valid to improve BER performance even with the imperfect CSI. For example, at a BER of $10^{-3}$, we can see that there are $4 \mathrm{~dB}$ gains for the modified system over the traditional one in the 1st detection iteration under the condition of imperfect CSI, that is, $\sigma_{e}^{2}=0.01$. Moreover, the BER results 
are quickly lowered with the increasing detection iterations. For instance, at a BER of $10^{-3}$, there are $2.5 \mathrm{~dB}$ extra gains attained for the modified system, where BF-TPA scheme is adopted through $3 \mathrm{rd}$ iterative detection under the condition of $\sigma_{e}^{2}=0.01$, which indicates that the proposed iterative detection scheme is an effective means to enhance the BER performance further even with imperfect CSI.

Figure 8 gives the BER performance of the modified Turbo-BLAST system with different number of active users under the condition of imperfect CSI $\left(\sigma_{e}^{2}=0.01\right)$, where $U$ denotes the number of active users and $I$ denotes the number of iterative detection. Figure 8 shows that at the same inaccuracy of channel estimation and for the identical number of iterative detection, the BER performance gets worse for large number of active users. For example, at a BER of $10^{-4}$, comparing the system that has sixty-four active users with the one having only one active user, there is $4.5 \mathrm{~dB}$ degradation of $E_{b} / N_{0}$. This is because the variance of each components of the equivalent noise is $U n_{T} \sigma_{e}^{2} / L_{S}+\sigma_{n}^{2}$; when the multiuser system was with more active users; the variance is approximately linear to the number of active users, so the BER results get worse with the increase of the number of active users.

\section{Conclusions}

In this paper, we first introduce a CDMA-based multiuser Turbo-BLAST system model, based on the imperfect channel model, the equivalent system model, and the variance of the equivalent noise. In order to maximize the capacity performance of the desired user, the SVD-assisted beamforming and power allocation strategy are theoretically obtained and the Lagrange multiplier method is then employed to find the optimized power allocation matrix, which is subject to the total transmit power constraint. And then, to circumvent the complexity of multiuser detection, the suboptimal decorrelating scheme is proposed to decouple the detection of different users. Finally, the iterative detection technique is adopted after postprocessing to enhance the BER results further. The complexity of the suboptimal receivers is exponential only in the transmission rate, similarly to singleuser Turbo-BLAST receivers. Numerical results show that the newly introduced method is effective to enhance the capacity results and the BER performance of multiuser Turbo-BLAST systems in the presence of imperfect CSI.

\section{Acknowledgments}

This work is supported by National Natural Science Foundation of China (61172077), Doctoral Fund of Ministry of Education of China (20093218120021), and Fundamental Research Funds for the Central Universities (NS2012075, NP2011036). The authors would like to thank the anonymous reviewers for their valuable comments.

\section{References}

[1] G. J. Foschini and M. J. Gans, "On limits of wireless communications in a fading environment when using multiple antennas," Wireless Personal Communications, vol. 6, no. 3, pp. 315-335, 1998.

[2] A. A. Abouda and S. G. Häggman, "Effect of mutual coupling on capacity of mimo wireless channels in high SNR scenario," Progress in Electromagnetics Research, vol. 65, pp. 27-40, 2006.

[3] P. W. Wolniansky, G. J. Foschini, G. D. Golden, and R. A. Valenzuela, "V-BLAST: an architecture for realizing very high data rates over the rich-scattering wireless channel," in Proceedings of the URSI International Symposium on Signals, Systems, and Electronics (ISSSE '98), pp. 295-300, Pisa, Italy, October 1998.

[4] M. Sellathurai and S. Haykin, "Turbo-BLAST for wireless communications: theory and experiments," IEEE Transactions on Signal Processing, vol. 50, no. 10, pp. 2538-2546, 2002.

[5] X. Chen, X. Yu, and D. Xu, "SVD-aided power allocation and iterative detection scheme for turbo-BLAST system with imperfect channel state information," International Journal of Antennas and Propagation, vol. 2011, Article ID 505606, pp. $1-8,2011$.

[6] R. L. Pickholtz, D. L. Schilling, and L. B. Milstein, "Theory of spread-spectrum communications-a tutorial," IEEE Transactions on Communications, vol. 30, no. 5, pp. 855-884, 1982.

[7] R. Lupas and S. Verdú, "Linear multiuser detectors for synchronous code-division multiple-access channels," IEEE Transactions on Information Theory, vol. 35, pp. 123-136, 1989.

[8] D. Tse and O. Zeitouni, "Performance of linear multiuser receivers in random environments," IEEE Transactions on Information Theory, vol. 46, no. 1, pp. 171-188, 2000.

[9] T. J. Willink, "Efficient adaptive SVD algorithm for MIMO applications," IEEE Transactions on Signal Processing, vol. 56, no. 2, pp. 615-622, 2008.

[10] W. Liu, L. L. Yang, and L. Hanzo, "SVD aided joint transmitter and receiver design for the uplink of multiuser detection assisted MIMO systems," in Proceedings of the IEEE International Conference on Communications (ICC '08), pp. 40954099, Beijing, China, May 2008.

[11] D. P. Palomar and J. R. Fonollosa, "Practical algorithms for a family of waterfilling solutions," IEEE Transactions on Signal Processing, vol. 53, no. 2, pp. 686-695, 2005.

[12] A. Yoshimoto and T. Hattori, "Area coverage of a multi-link MIMO system with water filling power allocation strategy," in Proceedings of the 66th IEEE Vehicular Technology Conference (VTC '07) Fall, pp. 1137-1141, Baltimore, Md, USA, October 2007.

[13] F. Wang and Z. Liu, "Adaptive water-filling power control for wireless communications networks," IEEE Communications Letters, vol. 12, no. 10, pp. 737-739, 2008.

[14] M. Brehler and M. K. Varanasi, "Coherent multiuser spacetime communications: optimum receivers and signal design," in Proceedings of the 35th Annual Conference Information Science System, pp. 372-378, Baltimore, Md, USA, 2001.

[15] M. Brehler and M. K. Varanasi, "Noncoherent multiuser space-time communications: optimum receivers and signal design," in Proceedings of the 35th Annual Conference Information Science System, pp. 379-383, Baltimore, Md, USA, 2001.

[16] H. B. Li and J. Li, "Differential and coherent decorrelating multiuser receivers for space-time-coded CDMA systems," IEEE Transactions on Signal Processing, vol. 50, no. 10, pp. 2529-2537, 2002.

[17] S. L. Zhou and G. B. Giannakis, "How accurate channel prediction needs to be for transmit-beamforming with adaptive modulation over Rayleigh MIMO channels?" IEEE 
Transactions on Wireless Communications, vol. 3, no. 4, pp. 1285-1294, 2004.

[18] I. E. Telatar, "Capacity of multi-antenna Gaussian channels," European Transactions on Telecommunications, vol. 10, no. 6, pp. 585-595, 1999. 

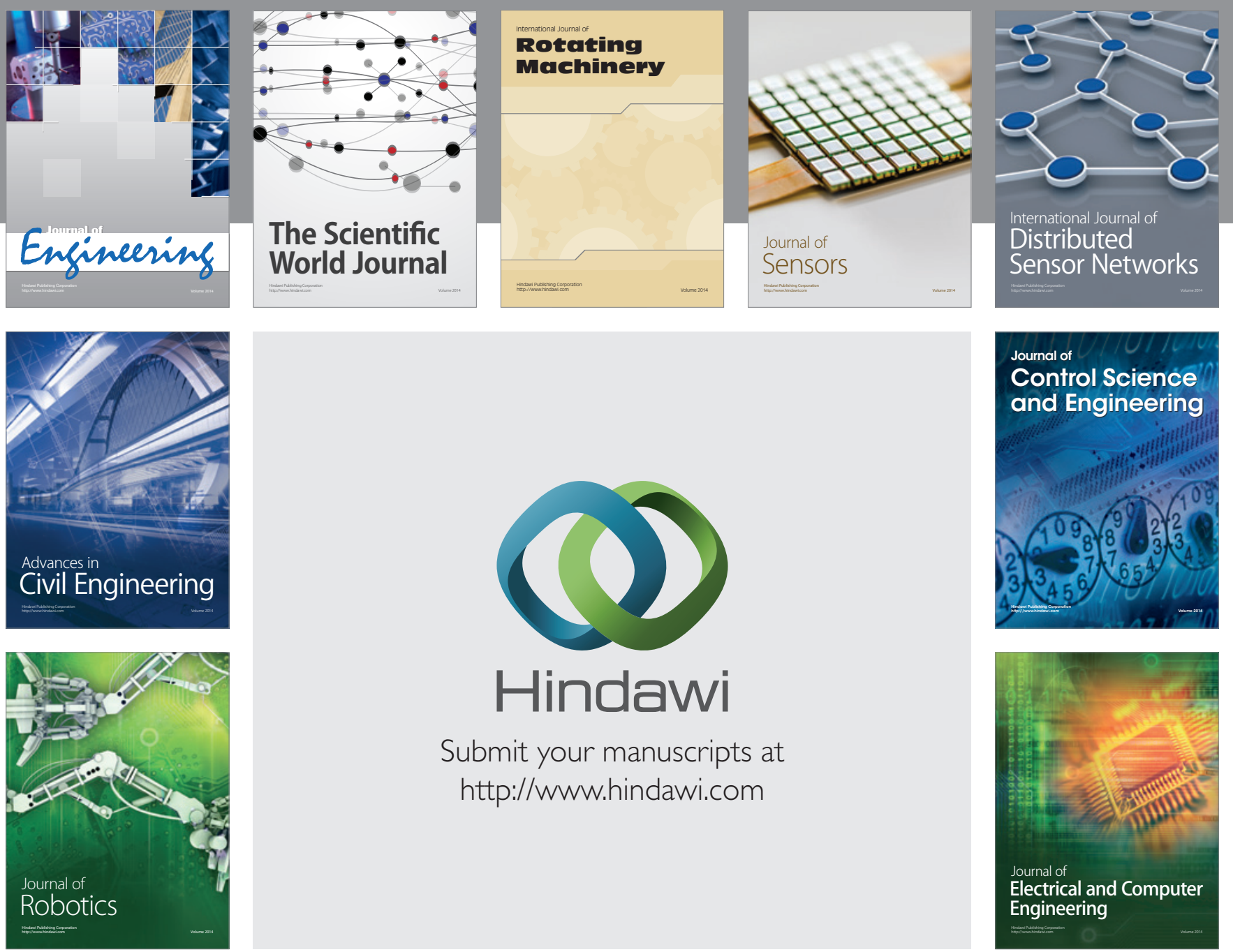

Submit your manuscripts at

http://www.hindawi.com
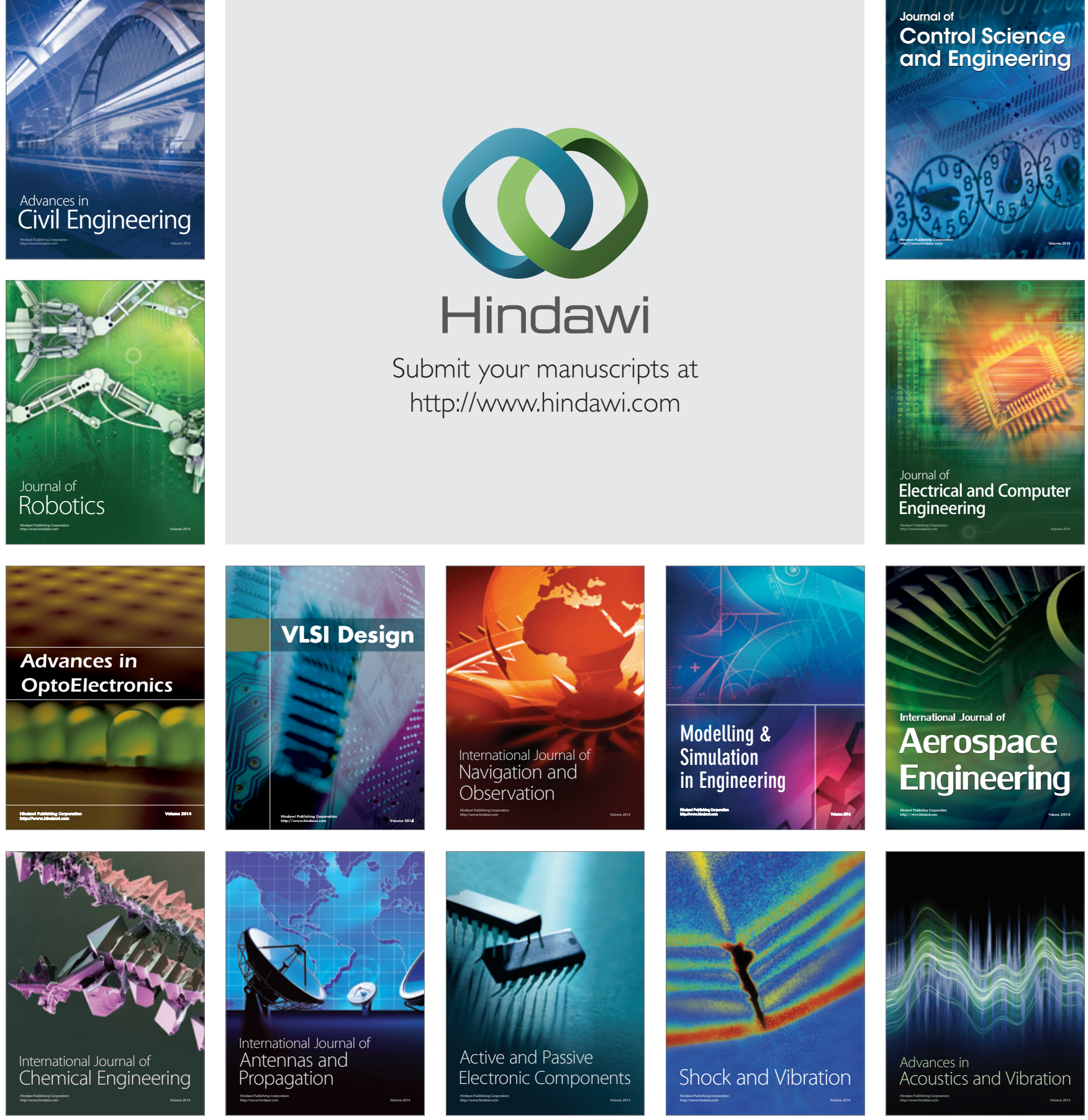